\title{
FINITE DIFFERENCE PRECONDITIONING FOR SOLVING ORTHOGONAL COLLOCATION EQUATIONS FOR BOUNDARY VALUE PROBLEMS*
}

\author{
WEIWEI SUN ${ }^{\dagger}$, WEIZHANG HUANG ${ }^{\ddagger}$, AND ROBERT D. RUSSELL ${ }^{\S}$
}

\begin{abstract}
A technique to construct a low-order finite difference preconditioner for solving orthogonal collocation equations for boundary value problems is presented. It is shown numerically and theoretically that the spectral condition numbers of the preconditioned collocation matrices are bounded by constants independent of the number of mesh nodes when certain exact low-order finite difference preconditionings are used. Preconditioners based on incomplete LU factorization are also discussed. Numerical experiments show the efficiency and robustness of the preconditioning.
\end{abstract}

Key words. collocation, preconditioning, boundary value problem

AMS subject classifications. $65 \mathrm{~N} 22,65 \mathrm{~N} 35,65 \mathrm{~L} 60$

1. Introduction. In this paper, we shall consider orthogonal collocation methods for solving elliptic differential equations. These methods have been extensively investigated in the last 20 years due to their ease of implementation and high-order accuracy (see [RS72], [DS73], and [PR76]). An apparent disadvantage of orthogonal collocation lies in the fact that a collocation matrix is generally nonsymmetric, even for a Poisson equation with a uniform mesh. Gauss elimination has often been used for solving the collocation equations, and its cost has restricted the applicability of the methods.

In recent years, some efforts in solving the collocation equations by alternative methods have been made. A so-called alternating direction collocation (ADC) or generalized implant (ADI) method was introduced by Hayes [Hay80] and Hayes, Pinder, and Celia [HPC81] for solving a parabolic problem. A full theory for convergence of ADC on a uniform mesh is given for the model problem $\Delta u=f$ in a rectangular domain by Dyksen [Dyk87]. Significantly, a closed form for the eigenvalues and their associated eigenfunctions is also given. Cooper and Prenter [CP91] give a complete theory for convergence of ADC applied to a large class of linear separable elliptic partial differential equations (PDEs) on a rectangular domain. Bialecki [Bia91] considers the problem of determining optimal acceleration parameters for ADC by using the Jordan acceleration technique. Besides ADC, a fast direct method (FFT) has been developed for solving $\Delta u=f$ on a uniform mesh in [SZ89] and [BFB92]. In [Sun94a], a class of block stationary (standard) methods are applied to second-order separable elliptic PDEs and their convergence is proven. However, all of the above methods are only applicable for linear separable and self-adjoint elliptic PDEs.

It has been well demonstrated that when solving PDEs a significant improvement in convergence of conventional iterative methods can be gained by using preconditioning techniques. They have been investigated extensively for finite difference, finite element, and spectral methods; e.g., see [Ors80], [Eva83], [Won87], [CE88], and [CHQZ88]. For spectral methods, a low-order finite difference approximation or its incomplete LU factorization can

${ }^{*}$ Received by the editors June 1, 1993; accepted for publication (in revised form) January 15, 1995. This work was supported in part by Natural Science and Engineering Research Council of Canada grant A8781.

${ }^{\dagger}$ Department of Mathematics and Statistics, Simon Fraser University, Burnaby, British Columbia V5A 1S6, Canada. Current address: School of Mathematical Sciences, Australian National University, Canberra, ACT 0200 Australia (weiwei@maths.anu.edu.au).

$\ddagger$ Department of Mathematics and Statistics, Simon Fraser University, Burnaby, British Columbia V5A 1S6, Canada. Current address: Department of Mathematics, University of Kansas, Lawrence, Kansas 66045 (whuang@ math.ukans.edu).

$\S$ Department of Mathematics and Statistics, Simon Fraser University, Burnaby, British Columbia V5A 1S6, Canada (rdr@cs.sfu.ca). 
often be proven to be an efficient preconditioning technique [Ors80]. Recently, for finite element and finite difference methods, some theoretical analysis applicable to a large class of elliptic PDEs has been carried out in [MP90] and [JMPW92]. However, to our knowledge preconditioning has not been applied in the orthogonal collocation context. The objective of this paper is to present a technique to construct such a finite difference preconditioning. A major difficulty lies in the fact that the unknowns in the Hermite collocation approximation involve both the variable $u$ and its derivatives, while those in the standard finite difference approximation contain only the variable $u$.

An outline of the paper is as follows. In $\S 2$, we introduce some notation and give a brief description of the orthogonal Hermite cubic collocation method. In $\S 3$, a technique to construct a finite difference preconditioning is presented for one- and two-dimensional (1D and 2D) model problems. Several versions of the preconditioning are discussed. In $\S 4$, we show that for the model problems with periodic boundary conditions and a uniform mesh, the condition number of a preconditioned system is bounded by a constant independent of the number of mesh nodes. Numerical results supporting this theory and showing the efficacy of the preconditioning methods are presented in $\S 5$. Section 6 contains conclusions and additional comments.

2. Orthogonal collocation methods. Consider the problem

$$
L u:=-u^{\prime \prime}+\alpha u=f(x), \quad x \in(0,1)
$$

with the Dirichlet boundary conditions $u(0)=u(1)=0$ or the periodic boundary conditions $u(0)=u(1)$ and $u^{\prime}(0)=u^{\prime}(1)$. Given a partition $\Pi_{N}=\left\{x_{i}\right\}_{i=0}^{N}$ on $[0,1]$ such that

$$
0=x_{0}<x_{1}<\cdots<x_{N}=1,
$$

the Hermite cubic collocation approximation $v$ is defined as follows:

$$
v(x)=\xi_{1}(s) u_{i}+h_{i} \xi_{2}(s) u_{i}^{\prime}+\xi_{1}(1-s) u_{i+1}-h_{i} \xi_{2}(1-s) u_{i+1}^{\prime}, \quad s \in[0,1]
$$

on each element $\left[x_{i}, x_{i+1}\right](i=0, \ldots, N-1)$, where $s=\left(x-x_{i}\right) / h_{i}, h_{i}=x_{i+1}-x_{i}, u_{i}$ and $u_{i}^{\prime}$ denote approximations to $u\left(x_{i}\right)$ and $u^{\prime}\left(x_{i}\right)$, respectively, and $\xi_{1}(s)$ and $\xi_{2}(s)$ are the so-called shape functions for 1D Hermite cubic interpolation defined by

$$
\begin{aligned}
& \xi_{1}(s)=(1+2 s)(1-s)^{2} \\
& \xi_{2}(s)=s(1-s)^{2}
\end{aligned}
$$

Denoting the collocation points by $\Pi_{C O L}:=\left\{x_{j}^{*}\right\}_{j=0}^{2 N+1}$, where

$$
\begin{array}{ll}
x_{0}^{*}=x_{0}-\sigma\left(x_{1}-x_{0}\right), & \\
x_{2 i+1}^{*}=x_{i}+\sigma\left(x_{i+1}-x_{i}\right), & i=0, \ldots, N-1, \\
x_{2 i+2}^{*}=x_{i}+(1-\sigma)\left(x_{i+1}-x_{i}\right), & i=0, \ldots, N-1, \\
x_{2 N+1}^{*}=x_{N}+\sigma\left(x_{N}-x_{N-1}\right), &
\end{array}
$$

with $\sigma:=\frac{1}{2}\left(1-\frac{1}{\sqrt{3}}\right)$ and $1-\sigma$ (the Gaussian points on $\left.(0,1)\right)$, the orthogonal Hermite cubic collocation approximation $v(x)$ of (2.1) is defined by the collocation equations

$$
L v\left(x_{2 i+l}^{*}\right)=-v^{\prime \prime}\left(x_{2 i+l}^{*}\right)+\alpha v\left(x_{2 i+l}^{*}\right)=f\left(x_{2 i+l}^{*}\right), \quad l=1,2 ; i=0, \ldots, N-1,
$$


and boundary conditions $v\left(x_{0}\right)=v\left(x_{2 N+1}\right)=0$. This can be rewritten in the matrix form

$$
A_{C O L} v:=A_{C} v+\alpha B_{C} v=f,
$$

where

$$
\begin{aligned}
& v=\left[u_{0}^{\prime}, u_{1}, u_{1}^{\prime}, \ldots, u_{N-1}, u_{N-1}^{\prime}, u_{N}^{\prime}\right]^{T}, \\
& f=\left[f\left(x_{1}^{*}\right), \ldots, f\left(x_{2 N}^{*}\right)\right]^{T} .
\end{aligned}
$$

The matrices $A_{C}$ and $B_{C}$ can be regarded as collocation approximations to the second derivative and identity operators, respectively, and are neither symmetric nor diagonally dominant. This is why, although some iterative and fast FFT-like methods have been developed recently for solving many PDE systems, Gauss elimination has predominated in the general collocation matrix context.

3. Finite difference preconditioning. In this section, we present a technique to construct a finite difference preconditioning for the orthogonal collocation system. For simplicity, the technique is illustrated for orthogonal Hermite cubic collocation applied to Dirichlet and periodic problems for 1D and 2D Poisson equations. However, as will be evident, the technique may be applied for more general collocation methods and boundary value problems.

3.1. 1D case. We consider the 1D Dirichlet model problem (2.1). The collocation approximation contains $2(N+1)$ unknowns, and we consider a finite difference approximation which involves the same number of unknowns. A natural way to construct such an approximation is to use centered differences based on the collocation points $\left\{x_{j}^{*}, j=0, \ldots, 2 N+1\right\}$. That is, use

$$
-\frac{2}{x_{j+1}^{*}-x_{j-1}^{*}}\left[\frac{w_{j+1}-w_{j}}{x_{j+1}^{*}-x_{j}^{*}}-\frac{w_{j}-w_{j-1}}{x_{j}^{*}-x_{j-1}^{*}}\right]+\alpha w_{j}=f\left(x_{j}^{*}\right), \quad j=1,2, \ldots, 2 N
$$

supplemented with the boundary conditions $w_{0}=w_{2 N+1}=0$, where $w_{j}$ denotes the finite difference approximation of $u\left(x_{j}^{*}\right)$. In matrix form this is

$$
A_{F} w=f
$$

where $f$ is defined by (2.6) and

$$
w=\left[w_{1}, \ldots, w_{2 N}\right]^{T} .
$$

Since $w$ and $v$ approximate different quantities, $A_{F}$ cannot be expected to be a suitable preconditioner for $A_{C O L}$. However, a relation between these two sets of unknowns is easily found using (2.2), viz., we have

$$
\begin{gathered}
v\left(x_{2 i+1}^{*}\right)=\xi_{1}(\sigma) u_{i}+h_{i} \xi_{2}(\sigma) u_{i}^{\prime}+\xi_{1}(1-\sigma) u_{i+1}-h_{i} \xi_{2}(1-\sigma) u_{i+1}^{\prime}, \\
v\left(x_{2 i+2}^{*}\right)=\xi_{1}(1-\sigma) u_{i}+h_{i} \xi_{2}(1-\sigma) u_{i}^{\prime}+\xi_{1}(\sigma) u_{i+1}-h_{i} \xi_{2}(\sigma) u_{i+1}^{\prime}, \\
i=0,1, \ldots, N-1 .
\end{gathered}
$$

Both $v\left(x_{j}^{*}\right)$ and $w_{j}$ are approximations to $u\left(x_{j}^{*}\right)$, and if $v\left(x_{j}^{*}\right)$ is replaced by $w_{j}$ we obtain

$$
w=B_{C} v .
$$

Substituting (3.5) into (2.5) gives

$$
A_{C O L} B_{C}^{-1} w=f
$$


TABLE 1

Condition numbers of the collocation and preconditioned matrices for the $1 D$ model problem.

\begin{tabular}{|c|c|cc|cc|c|c|}
\hline MESH NO. & $\mathrm{N}$ & $\begin{array}{c}\kappa_{1}\left(A_{C O L}\right) \\
\text { Dirichlet }\end{array}$ & $\begin{array}{c}\kappa_{1}\left(T_{1}\right) \\
(\alpha=0)\end{array}$ & $\begin{array}{c}\kappa_{1}\left(A_{C O L}\right) \\
\text { Periodic }\end{array}$ & $\begin{array}{c}\kappa_{1}\left(T_{1}\right) \\
(\alpha=1)\end{array}$ & $h_{1}^{*}$ & $h_{2}^{*}$ \\
\hline \multirow{3}{*}{$\begin{array}{c}1 \\
\text { uniform }\end{array}$} & 8 & $0.265 \mathrm{E} 2$ & 3.168 & $0.239 \mathrm{E} 3$ & 2.196 & 1.0 & 1.0 \\
\cline { 2 - 8 } & 16 & $0.983 \mathrm{E} 2$ & 3.168 & $0.883 \mathrm{E} 3$ & 2.196 & 1.0 & 1.0 \\
\cline { 2 - 8 } & 32 & $0.366 \mathrm{E} 3$ & 3.168 & $0.335 \mathrm{E} 4$ & 2.196 & 1.0 & 1.0 \\
\hline \multirow{3}{*}{2} & 8 & $0.159 \mathrm{E} 4$ & 3.123 & $0.743 \mathrm{E} 4$ & $0.903 \mathrm{E} 1$ & 4.0 & 49.0 \\
\cline { 2 - 8 } & 16 & $0.223 \mathrm{E} 5$ & 3.144 & $0.116 \mathrm{E} 6$ & $0.182 \mathrm{E} 2$ & 4.0 & 225.0 \\
\cline { 2 - 8 } & 32 & $0.326 \mathrm{E} 6$ & 3.156 & $0.186 \mathrm{E} 7$ & $0.364 \mathrm{E} 2$ & 4.0 & 961.0 \\
\hline \multirow{3}{*}{3} & 8 & $0.753 \mathrm{E} 7$ & 3.743 & $0.310 \mathrm{E} 8$ & $0.205 \mathrm{E} 4$ & 16.0 & 2401.0 \\
\cline { 2 - 8 } & 16 & $0.164 \mathrm{E} 10$ & 3.816 & $0.772 \mathrm{E} 10$ & $0.188 \mathrm{E} 5$ & 16.0 & 50625.0 \\
\cline { 2 - 8 } & 32 & $0.367 \mathrm{E} 12$ & 3.854 & $0.171 \mathrm{E} 13$ & $0.162 \mathrm{E} 6$ & 16.0 & 923521.0 \\
\hline \multirow{3}{*}{$\beta=10$} & 8 & $0.119 \mathrm{E} 4$ & 3.743 & $0.156 \mathrm{E} 5$ & 3.162 & 8.0 & 10.0 \\
\cline { 2 - 8 } & 16 & $0.595 \mathrm{E} 4$ & 3.176 & $0.451 \mathrm{E} 5$ & 3.161 & 8.0 & 10.0 \\
\cline { 2 - 8 }$\beta=100$ & 32 & $0.265 \mathrm{E} 5$ & 3.168 & $0.179 \mathrm{E} 6$ & 3.161 & 8.0 & 10.0 \\
\cline { 2 - 8 } & 8 & $0.104 \mathrm{E} 6$ & 25.08 & $0.114 \mathrm{E} 7$ & 25.11 & 80.0 & 100.0 \\
\cline { 2 - 8 } & 16 & $0.600 \mathrm{E} 6$ & 25.02 & $0.447 \mathrm{E} 7$ & 25.11 & 80.0 & 100.0 \\
\cline { 2 - 8 }$\beta=1000$ & 32 & $0.265 \mathrm{E} 7$ & 25.09 & $0.179 \mathrm{E} 8$ & 25.11 & 80.0 & 100.0 \\
\cline { 2 - 8 } & 8 & $0.118 \mathrm{E} 8$ & 244.7 & $0.115 \mathrm{E} 9$ & 245.8 & 800.0 & 1000.0 \\
\cline { 2 - 8 } & 16 & $0.599 \mathrm{E} 8$ & 244.9 & $0.452 \mathrm{E} 9$ & 245.8 & 800.0 & 1000.0 \\
\hline \multirow{3}{*}{4} & $0.264 \mathrm{E} 9$ & 244.9 & $0.179 \mathrm{E} 10$ & 245.8 & 800.0 & 1000.0 \\
\hline
\end{tabular}

Fig. 1. $1 D$ Mesh 4 for $N=8$.

Now (3.6) has the same unknowns as (3.2), which motivates using $A_{F}$ as our preconditioner for $A_{C O L} B_{C}^{-1}$. The periodic problem can be considered similarly.

The accuracy of this preconditioner has been examined for several examples. Throughout, the numerical experiments are performed on a Sun SPARC +1 in double precision, using standard IMSL routines for solving the linear systems and eigenvalue problems.

In Table 1 , the condition numbers of $A_{C O L}$ and $T_{1}:=A_{F}^{-1}\left(A_{C O L} B_{C}^{-1}\right)$ for Dirichlet boundary conditions with $\alpha=0$ and periodic boundary conditions with $\alpha=1$ are listed. The condition number used here is defined as

$$
\kappa_{1}(T)=\frac{\max _{i}\left|\lambda_{i}(T)\right|}{\min _{i}\left|\lambda_{i}(T)\right|},
$$

and the meshes are the following:

- 1D Mesh 1: uniform mesh, $x_{i}=\frac{i}{N}, i=0,1, \ldots, N$;

- 1D Mesh 2: $x_{i}=\left(\frac{i}{N}\right)^{2}, i=0,1, \ldots, N$;

- 1D Mesh 3: $x_{i}=\left(\frac{i}{N}\right)^{4}, i=0,1, \ldots, N$;

- 1D Mesh 4: a uniform mesh consisting of $N-3$ nodes, with four additional local uniform nodes close to $x=0.5$ (see Figure 1). The ratio of these two spacings is denoted by $\beta$.

The two quantities

$$
\begin{aligned}
& h_{1}^{*}=\max _{i}\left\{\max \left\{\frac{h_{i}}{h_{i+1}}, \frac{h_{i+1}}{h_{i}}\right\}\right\}, \\
& h_{2}^{*}=\frac{\max h_{i}}{\min h_{i}},
\end{aligned}
$$

the so-called local mesh ratio, and global mesh ratio are used to measure the smoothness of a given mesh. In Figures 2 and 3 , we plot the distributions of eigenvalues of $A_{C O L}$ and $T_{1}$ 

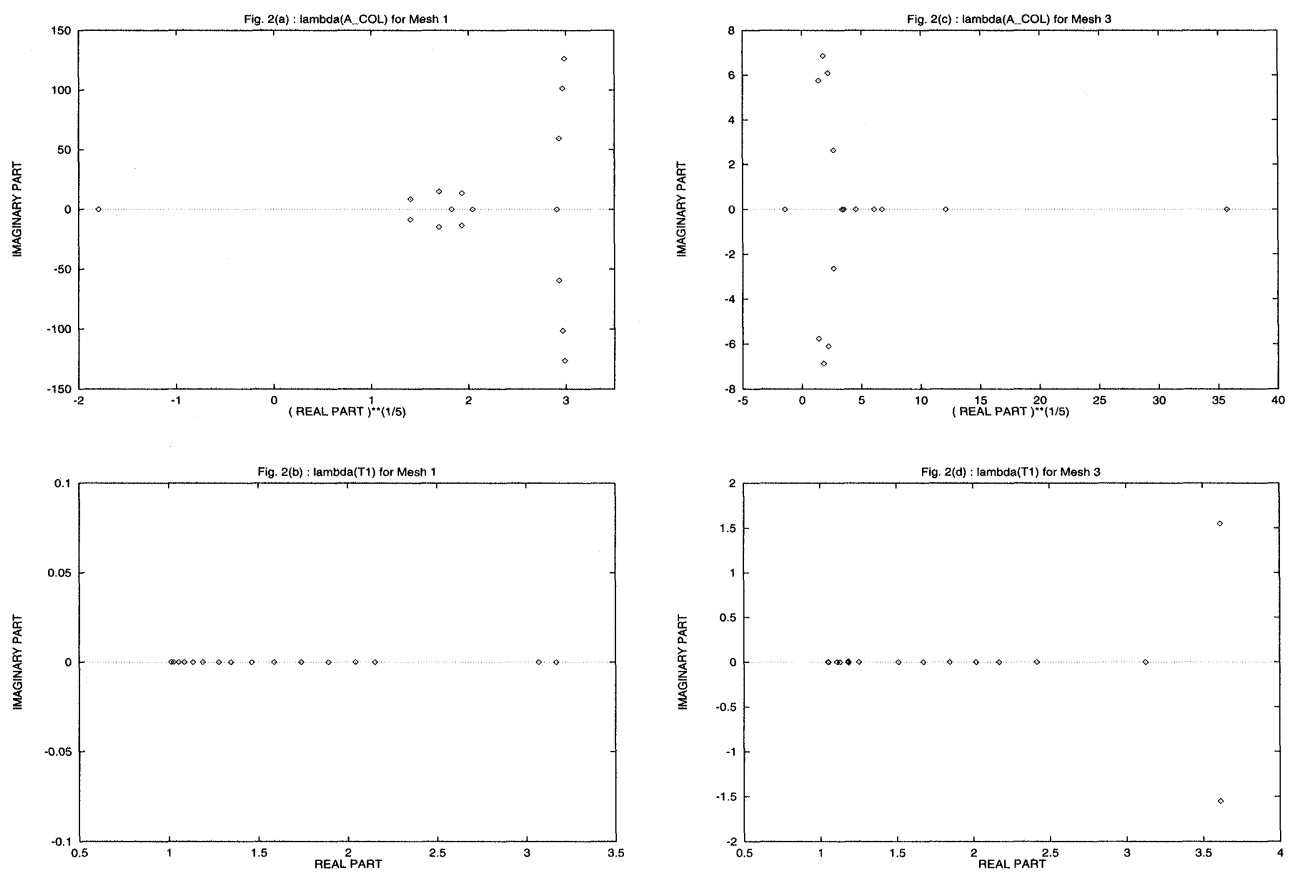

FIG. 2. Eigenvalues for the $1 D$ model problem with Dirichlet boundary conditions $(N=8, \alpha=0)$.
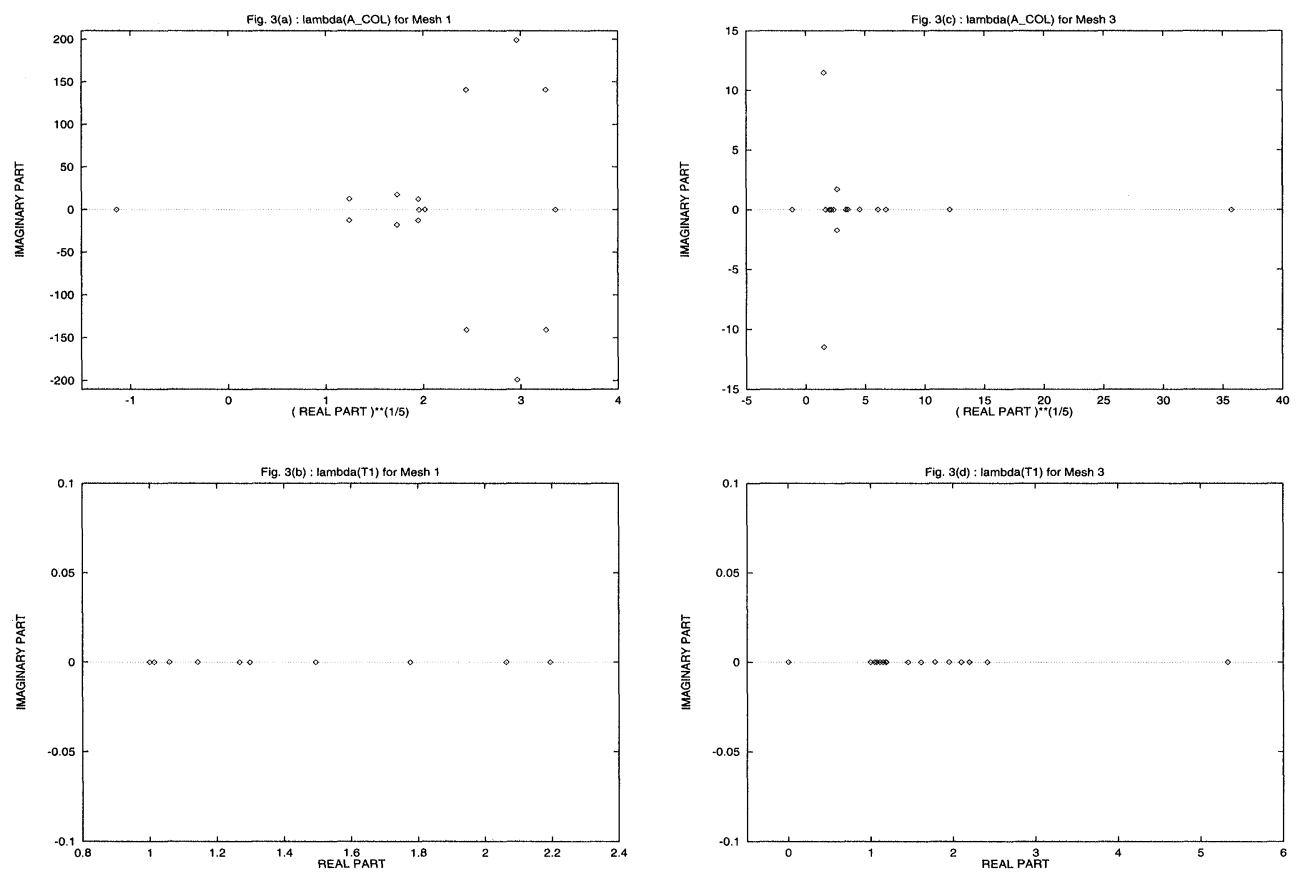

FIG. 3. Eigenvalues for the $1 D$ model problem with periodic boundary conditions $(N=8, \alpha=1)$.

for 1D Meshes 1 and 3 and $N=8$ with Dirichlet and periodic boundary conditions. Several observations can be made. 
Many of the eigenvalues of $A_{C O L}$ are complex. $A_{C O L}$ is indefinite, as its eigenvalues have both positive and negative real parts. This illustrates why conventional iterative methods, such as Jacobi, Gauss-Seidel and SOR, are divergent when they are applied to the collocation equations (2.5). The condition number of $A_{C O L}$ is quite sensitive to the smoothness of the mesh used.

Since both $A_{C O L} B_{C}^{-1}$ and $A_{F}$ are symmetric for 1D Mesh 1 [Bia91], the eigenvalues of $T_{1}$ are real and positive. In the case of Dirichlet boundary conditions, the condition numbers of $T_{1}$ are less sensitive than $A_{C O L}$ to the smoothness of the mesh. For a smooth or a moderately nonsmooth mesh ( $h_{1}^{*}$ is not large and the spacing does not have a steep change, such as 1D Meshes $1,2,3$, and 4 with $\beta=10), \kappa_{1}\left(T_{1}\right)$ is small. Even for a fairly nonsmooth mesh (such as 1D Mesh 4 with $\beta=100$ or 1000 , whose spacing has a steep jump near $x=0.5$ ), $\kappa_{1}\left(T_{1}\right)$ is much smaller than $\kappa_{1}\left(A_{C O L}\right)$. For all meshes, the norms of the eigenvalues of the preconditioned matrix are bounded below by one and above by a constant which is independent of $N$. For example, the upper bound is about 3.17 for 1D Mesh 1, 3.16 for 1D Mesh 2, and 3.86 for 1D Mesh 3 with Dirichlet boundary conditions. A similar observation can be made for the periodic boundary value problem except for Mesh 2 and Mesh 3 which are not suitable to this case.

Thus $A_{F}$ is a good preconditioner for $A_{C O L} B_{C}^{-1}$. The preconditioned matrix has a desirable distribution of eigenvalues for smooth and moderately nonsmooth meshes. Some of these numerical observations will be confirmed theoretically in $\S 4$. In $\S 5$ it is demonstrated that fast convergence can be gained when a conventional iterative method, such as Richardson and conjugate gradient iteration, is applied to the preconditioned system.

3.2. 2D case. Now we illustrate the preconditioning technique for the more interesting case of a 2D Helmholtz equation

$$
-\Delta u+\alpha u=f(x, y), \quad(x, y) \in \Omega
$$

with Dirichlet or periodic boundary conditions on $\partial \Omega$ where $\Omega=(0,1) \times(0,1)$. Four meshes are used:

$$
\text { 2D Mesh i }:=1 \text { D Mesh i } \times 1 \text { Desh i, i = 1, 2, 3, } 4 \text {. }
$$

On $\Pi_{C O L} \times \Pi_{C O L}$, the orthogonal Hermite bicubic collocation and five-point centered finite difference approximations to (3.8) are obtained in the same way as in the $1 \mathrm{D}$ case. They are also denoted (using the natural ordering) by $A_{C O L} v=f$ and $A_{F} w=f$. A relation between the collocation and finite difference approximations can be obtained and expressed by

$$
w=\left(B_{C} \otimes B_{C}\right) v
$$

where $B_{C}$ is defined in (2.5) and $\otimes$ denotes the Kronecker tensor product. Using $A_{F}$ as a preconditioner, the resulting preconditioned collocation system is

$$
T_{2} w=f
$$

where

$$
T_{2}:=A_{F}^{-1}\left(A_{C O L}\left(B_{C} \otimes B_{C}\right)^{-1}\right) .
$$

The condition numbers of $A_{C O L}$ and $T_{2}$ are shown in Table 2 for both Dirichlet boundary conditions with $\alpha=0$ and periodic boundary conditions with $\alpha=1$. These results confirm the observations made in the 1D case. It is interesting to notice that for Mesh 4 the condition number again increases linearly with the measure $\beta$ of its smoothness for both Dirichlet and periodic boundary conditions. 
TABLE 2

Condition numbers of the collocation and preconditioned matrices for the $2 D$ model problems.

\begin{tabular}{|c|c|c|c|c|c|c|c|}
\hline MESH NO. & $\mathrm{N}$ & $\begin{array}{c}\kappa_{1}\left(A_{C O L}\right) \\
\text { Dirichlet }\end{array}$ & $\begin{array}{l}\kappa_{1}\left(T_{2}\right) \\
(\alpha=0)\end{array}$ & $\begin{array}{c}\kappa_{1}\left(A_{C O L}\right) \\
\text { Periodic }\end{array}$ & $\begin{array}{l}\kappa_{1}\left(T_{2}\right) \\
(\alpha=1)\end{array}$ & $h_{1}^{*}$ & $h_{2}^{*}$ \\
\hline \multirow{3}{*}{$\begin{array}{c}1 \\
\text { uniform }\end{array}$} & 4 & $0.139 \mathrm{E} 3$ & 3.125 & $0.920 \mathrm{E} 2$ & 2.194 & 1.0 & 1.0 \\
\hline & 8 & $0.583 \mathrm{E} 3$ & 3.128 & $0.399 \mathrm{E} 3$ & 2.196 & 1.0 & 1.0 \\
\hline & 16 & $0.239 \mathrm{E} 4$ & 3.128 & $0.165 \mathrm{E} 4$ & 2.196 & 1.0 & 1.0 \\
\hline \multirow[t]{3}{*}{2} & 4 & $0.215 \mathrm{E} 4$ & 3.045 & $0.494 \mathrm{E} 3$ & 4.531 & 4.0 & 9.0 \\
\hline & 8 & $0.351 \mathrm{E} 5$ & 3.082 & $0.697 \mathrm{E} 4$ & 9.059 & 4.0 & 49.0 \\
\hline & 16 & $0.563 \mathrm{E} 6$ & 3.135 & $0.132 \mathrm{E} 6$ & 18.17 & 4.0 & 225.0 \\
\hline \multirow[t]{3}{*}{3} & 4 & $0.566 \mathrm{E} 6$ & 3.557 & $0.111 \mathrm{E} 6$ & $0.201 \mathrm{E} 3$ & 16.0 & 81.0 \\
\hline & 8 & $0.145 \mathrm{E} 9$ & 3.622 & $0.258 \mathrm{E} 8$ & $0.206 \mathrm{E} 4$ & 16.0 & 2461.0 \\
\hline & 16 & $0.371 \mathrm{E} 11$ & 3.761 & $0.804 \mathrm{E} 10$ & $0.189 \mathrm{E} 5$ & 16.0 & 50625.0 \\
\hline \multirow{2}{*}{$\begin{array}{c}4 \\
\beta=10\end{array}$} & 8 & $0.554 \mathrm{E} 5$ & 3.743 & $0.224 \mathrm{E} 5$ & 3.238 & 8.0 & 10.0 \\
\hline & 16 & $0.221 \mathrm{E} 6$ & 3.193 & $0.709 \mathrm{E} 5$ & 3.234 & 8.0 & 10.0 \\
\hline \multirow{2}{*}{$\begin{array}{c}4 \\
\beta=100\end{array}$} & 8 & $0.552 \mathrm{E} 7$ & 25.08 & $0.237 \mathrm{E} 7$ & 25.85 & 80.0 & 100.0 \\
\hline & 16 & $0.221 \mathrm{E} 8$ & 25.71 & $0.740 \mathrm{E} 7$ & 25.80 & 80.0 & 100.0 \\
\hline \multirow{2}{*}{$\begin{array}{c}4 \\
\beta=1000\end{array}$} & 8 & $0.552 \mathrm{E} 9$ & 245.4 & $0.242 \mathrm{E} 9$ & 253.0 & 800.0 & 1000.0 \\
\hline & 16 & $0.221 \mathrm{E} 10$ & 251.5 & $0.761 \mathrm{E} 9$ & 252.5 & 800.0 & 1000.0 \\
\hline
\end{tabular}

3.3. Incomplete $L U$ factorization preconditioning. For $1 \mathrm{D}$ problems, finite difference preconditioning is a quite inexpensive part of the iterative scheme. In higher dimensions, however, inversion of these finite difference matrices can become relatively expensive and/or complicated. This disadvantage is overcome by using a multigrid method or an incomplete LU factorization technique. Here, for simplicity we restrict our attention to the latter and the approach for a Dirichlet problem.

If a five-point finite difference approximation to a 2D second-order elliptic differential equations is given by

$$
\left(A_{F} u\right)_{i, j}=S_{i, j} u_{i, j-1}+W_{i, j} u_{i-1, j}+C_{i, j} u_{i, j}+N_{i, j} u_{i, j+1}+E_{i, j} u_{i+1, j},
$$

then a five-diagonal incomplete LU factorization can be expressed by

$$
\begin{aligned}
& (L u)_{i, j}=c_{i, j} u_{i, j}+s_{i, j} u_{i, j-1}+w_{i, j} u_{i-1, j}, \\
& (U u)_{i, j}=u_{i, j}+n_{i, j} u_{i, j+1}+e_{i, j} u_{i+1, j},
\end{aligned}
$$

where

$$
\begin{aligned}
& s_{i, j}=S_{i, j}, \\
& w_{i, j}=W_{i, j}, \\
& c_{i, j}=C_{i, j}-w_{i, j} e_{i-1, j}-s_{i, j} n_{i, j-1}-\gamma\left(s_{i, j} e_{i, j-1}+w_{i, j} n_{i-1, j}\right), \\
& n_{i, j}=N_{i, j} / c_{i, j}, \\
& e_{i, j}=E_{i, j} / c_{i, j}
\end{aligned}
$$

(see [CHQZ88] and [Won87]). The choice $\gamma=0$ gives the standard incomplete LU factorization $H_{L U S}$ (ILU) and $\gamma=1$ gives the row sum equivalence version $H_{R S 5}$ (MILU [CE88]). Using these preconditionings for the $A_{F}$ factor, we have the two new preconditioned matrices

$$
T_{3}:=H_{R S 5}^{-1}\left(A_{C O L}\left(B_{C} \otimes B_{C}\right)^{-1}\right) \text { and } T_{4}:=H_{L U 5}^{-1}\left(A_{C O L}\left(B_{C} \otimes B_{C}\right)^{-1}\right) .
$$

Note that the LU factorizations of $B_{C}$ and $B_{C} \otimes B_{C}$ are inexpensive because of their sparse and banded structure. 
TABLE 3

Extreme eigenvalues of the $H_{R S 5}$ and $H_{L U 5}$ preconditioned matrices for the $2 D$ Laplacian operator with Dirichlet boundary conditions and $\alpha=0$.

\begin{tabular}{|c|c|ccc|ccc|}
\hline \multirow{3}{*}{ MESH NO. } & \multirow{2}{*}{$\mathrm{N}$} & \multicolumn{3}{|c|}{$T_{3}$} & \multicolumn{3}{|c|}{$T_{4}$} \\
\cline { 3 - 8 } & & $\min \left|\lambda_{i}\right|$ & $\max \left|\lambda_{i}\right|$ & $\max \left|\frac{\operatorname{Im}\left(\lambda_{i}\right)}{\operatorname{Re}\left(\lambda_{i}\right)}\right|$ & $\min \left|\lambda_{i}\right|$ & $\max \left|\lambda_{i}\right|$ & $\max \left|\frac{\operatorname{Im}\left(\lambda_{i}\right)}{\operatorname{Re}\left(\lambda_{i}\right)}\right|$ \\
\hline \multirow{3}{*}{$\begin{array}{c}1 \\
\text { uniform }\end{array}$} & 4 & 1.197 & 3.728 & 0.0 & 0.389 & 3.040 & 0.0 \\
\cline { 2 - 8 } & 8 & 1.089 & 5.014 & 0.0 & 0.119 & 3.037 & 0.0 \\
\cline { 2 - 8 } & 16 & 1.044 & 9.814 & 0.0 & 0.032 & 3.037 & 0.0 \\
\hline \multirow{2}{*}{2} & 4 & 1.133 & 3.365 & 0.121 & 0.590 & 3.047 & 0.106 \\
\cline { 2 - 8 } & 8 & 1.054 & 4.217 & 0.132 & 0.202 & 3.045 & 0.123 \\
\cline { 2 - 8 } & 16 & 1.025 & 5.894 & 0.163 & 0.054 & 3.073 & 0.141 \\
\hline \multirow{3}{*}{3} & 4 & 1.178 & 3.717 & 0.426 & 0.949 & 3.686 & 0.623 \\
\cline { 2 - 8 } & 8 & 1.075 & 3.717 & 0.429 & 0.426 & 3.686 & 0.434 \\
\cline { 2 - 8 } & 16 & 1.032 & 5.031 & 0.429 & 0.126 & 3.686 & 0.434 \\
\hline
\end{tabular}

Table 3 summarizes how the extreme eigenvalues of $T_{3}$ and $T_{4}$ depend on $N$ and the smoothness of the mesh. There are some differences from using an exact factorization of the original finite difference matrix. Roughly speaking, the incomplete LU case is somewhat better for the high-frequency components and the row sum equivalence alternative is more accurate for the low-frequency components, as can be seen from Table 3. In fact, the minimal eigenvalue of $T_{3}$ is nearly one, which implies that $H_{R S 5}$ and $A_{C O L}\left(B_{C} \otimes B_{C}\right)^{-1}$ have similar low-frequency components. On the other hand, the maximal eigenvalue of $T_{4}$ is bounded above by a constant independent of $N$, and $H_{L U 5}$ and $A_{C O L}\left(B_{C} \otimes B_{C}\right)^{-1}$ have similar high-frequency components. Unlike the exact finite difference preconditioning, the condition numbers of both $T_{3}$ and $T_{4}$ can increase slowly with $N$. Since convergence rates for most conventional iterative methods are strongly related to the condition number, the row sum equivalence version on this basis appears to be more suitable as a preconditioning. Other important indicators for the convergence of iterative methods are ratios of real parts and imaginary parts of eigenvalues, which are listed in the table. It is obvious that the maximal ratio is strongly dependent upon the smoothness of meshes.

A more accurate factorization can be achieved by including one extra nonzero diagonal in each of $L$ and $U$. The seven-diagonal $L U$ factorization is given by (see [Won87] and [CHQZ88])

$$
\begin{aligned}
& (L u)_{i, j}=c_{i, j} u_{i, j}+s_{i, j} u_{i, j-1}+w_{i, j} u_{i-1, j}+\tilde{e}_{i, j} u_{i+1, j-1}, \\
& (U u)_{i, j}=u_{i, j}+n_{i, j} u_{i, j+1}+e_{i, j} u_{i+1, j}+\tilde{w}_{i, j} u_{i-1, j+1},
\end{aligned}
$$

where the coefficients can be calculated from

$$
\begin{aligned}
& s_{i, j}=S_{i, j}, \\
& w_{i, j}=W_{i, j}-s_{i, j} \tilde{w}_{i, j-1}, \\
& \tilde{e}_{i, j}=-s_{i, j} e_{i, j-1}, \\
& c_{i, j}=C_{i, j}-w_{i, j} e_{i-1, j}-s_{i, j} n_{i, j-1}-\tilde{e}_{i, j} \tilde{w}_{i+1, j-1}-\gamma\left(e_{i+1, j-1} \tilde{e}_{i, j}+w_{i, j} \tilde{w}_{i-1, j}\right), \\
& n_{i, j}=N_{i, j} / c_{i, j}, \\
& e_{i, j}=\left(E_{i, j}-n_{i+1, j-1} \tilde{e}_{i, j}\right) / c_{i, j}, \\
& \tilde{w}_{i, j}=-w_{i, j} n_{i-1, j} / c_{i, j} .
\end{aligned}
$$

Now $\gamma=0$ gives the $H_{L U 7}$ version and $\gamma=1$ the $H_{R S 7}$ version. Table 4 shows the extreme 
TABLE 4

Extreme eigenvalues of the $H_{R S 7}$ and $H_{L U 7}$ preconditioned matrices for the $2 D$ Laplacian operator with Dirichlet boundary conditions and $\alpha=0$.

\begin{tabular}{|c|c|c|c|c|c|c|c|}
\hline \multirow[b]{2}{*}{ MESH NO. } & \multirow[b]{2}{*}{$\mathrm{N}$} & \multicolumn{3}{|c|}{$T_{5}$} & \multicolumn{3}{|c|}{$T_{6}$} \\
\hline & & $\min \left|\lambda_{i}\right|$ & $\max \left|\lambda_{i}\right|$ & $\max \left|\frac{\operatorname{Im}\left(\lambda_{i}\right)}{\operatorname{Re}\left(\lambda_{i}\right)}\right|$ & $\min \left|\lambda_{i}\right|$ & $\max \left|\lambda_{i}\right|$ & $\max \left|\frac{\operatorname{Im}\left(\lambda_{i}\right)}{\operatorname{Re}\left(\lambda_{i}\right)}\right|$ \\
\hline \multirow{3}{*}{$\begin{array}{c}1 \\
\text { uniform }\end{array}$} & 4 & 1.168 & 3.102 & 0.0 & 0.714 & 3.037 & 0.0 \\
\hline & 8 & 1.082 & 3.280 & 0.0 & 0.278 & 3.020 & 0.0 \\
\hline & 16 & 1.043 & 5.458 & 0.0 & 0.081 & 3.018 & 0.0 \\
\hline \multirow[t]{3}{*}{2} & 4 & 1.103 & 3.102 & 0.135 & 0.901 & 3.047 & 0.113 \\
\hline & 8 & 1.049 & 3.215 & 0.142 & 0.433 & 3.031 & 0.130 \\
\hline & 16 & 1.010 & 3.570 & 0.144 & 0.134 & 3.016 & 0.144 \\
\hline \multirow[t]{3}{*}{3} & 4 & 1.170 & 3.672 & 0.574 & 1.140 & 3.671 & 0.543 \\
\hline & 8 & 1.070 & 3.672 & 0.425 & 0.743 & 3.671 & 0.425 \\
\hline & 16 & 1.026 & 3.672 & 0.425 & 0.287 & 3.671 & 0.425 \\
\hline
\end{tabular}

eigenvalues of $T_{5}:=H_{R S 7}^{-1} A_{C O L}(B \otimes B)^{-1}$ and $T_{6}:=H_{L U 7}^{-1} A_{C O L}(B \otimes B)^{-1}$. Results are consistent with the observation concerning $T_{3}$ and $T_{4}$, but the condition numbers are smaller.

4. Condition number estimates. The numerical results in the previous sections illustrate that the eigenvalues of the preconditioned collocation systems are bounded below by one and above by a constant which is independent of the number of mesh points, except in the case of Mesh 2 and Mesh 3 with periodic boundary conditions. Here, we shall present a theoretical analysis of the preconditioned system. The discussion is restricted to the simple model problem (2.1) with periodic boundary conditions and a uniform collocation mesh (Mesh 1).

For a uniform collocation mesh $\left\{x_{i}\right\}_{i=0}^{2 N}$ a corresponding finite difference approximation for (2.1) with periodic boundary conditions is produced on the mesh $\left\{x_{i}^{*}\right\}_{i=0}^{4 N+1}$ defined in (2.3). Arranging the variables and equations to correspond to the node ordering $\left\{x_{0}^{*}, x_{2}^{*}, \ldots, x_{4 N}^{*}, x_{1}^{*}, \ldots, x_{4 N+1}^{*}\right\}$ and using the periodic boundary conditions $u\left(x_{0}^{*}\right)=u\left(x_{4 N}^{*}\right)$ and $u\left(x_{4 N+1}^{*}\right)=u\left(x_{1}^{*}\right)$, the global finite difference matrix is given by

$$
A_{F}=\left(\begin{array}{cc}
(b+\alpha) I & -C \\
-C^{T} & (b+\alpha) I
\end{array}\right),
$$

where $I$ is the $2 N \times 2 N$ identity matrix,

$$
C=\left(\begin{array}{cccc}
c & 0 & \cdots & a \\
a & c & \cdots & 0 \\
& \ddots & \ddots & \\
& & a & c
\end{array}\right)
$$

is a circulant matrix,

$$
\begin{gathered}
a=\frac{2}{h_{2}\left(h_{1}+h_{2}\right)}, \quad b=\frac{2}{h_{1} h_{2}}, \quad c=\frac{2}{h_{1}\left(h_{1}+h_{2}\right)}, \\
h_{1}=(1-2 \sigma) h, \quad h_{2}=2 \sigma h,
\end{gathered}
$$

and $h$ is the collocation mesh stepsize. The corresponding Hermite cubic collocation system is given by

$$
A_{C} u+\alpha B_{C} u=f
$$


or

$$
\left(A_{C} B_{C}^{-1}+\alpha I\right) \bar{u}=f
$$

where $\bar{u}=B_{C} u$. The objective of this section is to establish bounds on condition numbers of the matrix

$$
T=A_{F}^{-1}\left(A_{C} B_{C}^{-1}+\alpha I\right) .
$$

Our approach is an algebraic one.

4.1. Eigensystem of collocation matrix. The eigenvalues and eigenvectors for the finite difference matrix are well known, and the corresponding eigensystem for the Hermite collocation matrix has been analyzed by Dyksen [Dyk87]. He considers the generalized eigenvalue problem $-\bar{v}^{\prime \prime}=\lambda \bar{v}$ and the corresponding matrix eigenvalue problem

$$
A_{C} B_{C}^{-1} v=\lambda v
$$

with Dirichlet boundary conditions and gives an analytic expression of the eigenvalues. The problem with periodic boundary conditions also has been analyzed in [Sun94b], and these results can be summarized as follows:

LEMMA 4.1. Let $\left(A_{C}, B_{C}\right)$ denote a Hermite cubic collocation discretization for (2.1) with periodic boundary conditions. The eigenvalue problem $A_{C} B_{C}^{-1} v=\lambda u$ with $\alpha=0$ has $a$ complete set of eigenvectors for which the eigenvalues are nonnegative and are given by

$$
\lambda_{ \pm l}^{c}=\frac{-v \pm \sqrt{v^{2}-4 \mu \omega}}{-2 \mu}, \quad l=1, \ldots, 2 N-1, l \neq N, l \neq-N
$$

and

$$
\lambda_{-0}^{c}=\frac{24}{\left(1-4 \tau^{2}\right) h^{2}}, \quad \lambda_{0}^{c}=0, \quad \lambda_{-N}=\frac{12}{h^{2}}, \quad \lambda_{N}=\frac{9}{h^{2}},
$$

where

$$
\begin{aligned}
& \mu=h^{4}\left[\left(16 \tau^{4}-16 \tau^{2}+3\right) \rho-8 \tau^{2}+2\right], \\
& \nu=h^{2}\left[\left(-128 \tau^{2}+48\right) \rho+48\right], \\
& \omega=192 \rho, \\
& \rho=\tan ^{2}\left(\frac{\theta_{l}}{2}\right), \quad \theta_{l}=l \pi / N,
\end{aligned}
$$

and $\tau=\frac{1}{2 \sqrt{3}}\left(\tau=\frac{1}{2}-\sigma\right)$ for Gaussian collocation points. The corresponding eigenvectors are given by

$$
\begin{aligned}
& v_{l}=d_{l}\left(\begin{array}{c}
\left(-\frac{z_{l}}{2} \sin k \bar{\theta}_{l}-\tau h \cos k \bar{\theta}_{l}\right)_{k=0}^{2 N-1} \\
\left(-\frac{z_{l}}{2} \sin k \bar{\theta}_{l}+\tau h \cos k \bar{\theta}_{l}\right)_{k=0}^{2 N-1}
\end{array}\right), \quad l=-0,1 \leq|l| \leq N-1, \\
& v_{l}=d_{l}\left(\begin{array}{c}
\left(\frac{z_{l}}{2} \cos k \bar{\theta}_{l}-\tau h \sin k \bar{\theta}_{l}\right)_{k=0}^{2 N-1} \\
\left(\frac{z_{l}}{2} \cos k \bar{\theta}_{l}+\tau h \sin k \bar{\theta}_{l}\right)_{k=0}^{2 N-1}
\end{array}\right), \quad N+1 \leq|l| \leq 2 N-1,
\end{aligned}
$$




$$
v_{0}=\frac{1}{2}\left(\begin{array}{l}
e \\
e
\end{array}\right), \quad v_{N}=\frac{1}{2}\left(\begin{array}{c}
-e^{*} \\
-e^{*}
\end{array}\right) \quad \text { and } \quad v_{-N}=\frac{1}{2}\left(\begin{array}{c}
-e^{*} \\
e^{*}
\end{array}\right),
$$

where

$$
\begin{gathered}
z_{l}=\frac{4}{h}\left(-\frac{1}{\lambda_{l}}-\frac{\tau^{2} h^{2}}{6}+\frac{h^{2}}{8}\right) \tan \frac{\bar{\theta}_{l}}{2}, \\
\bar{\theta}_{l}=\left|\theta_{l}\right|, \quad \theta_{-0}=\theta_{0},
\end{gathered}
$$

$e=(1,1, \ldots, 1)^{T}, e^{*}=(1,-1,1,-1, \ldots, 1,-1)^{T} \in \mathbb{R}^{2 N}$ and $d_{l}$ is a constant

It is not difficult to obtain from Lemma 4.1 that

$$
\pi^{2}<\lambda_{l}^{c} \leq \frac{9}{h^{2}} \quad \text { and } \quad \frac{12}{h^{2}}<\lambda_{-l}^{c}<\lambda_{-0}=\frac{36}{h^{2}}, \quad l=1,2, \ldots, 2 N-1 .
$$

The following Lemma is a result of the fact $A_{C} B_{C}^{-1}$ is symmetric when Gaussian points are chosen as the collocation points [Ben91].

LEMMA 4.2. The eigenvectors $\left\{v_{l}\right\}_{l= \pm 0}^{ \pm(2 N-1)}$ defined in (4.10)-(4.12) form an orthogonal set under the inner product

$$
\langle u, v\rangle=\sum_{i} u_{i} v_{i}, \quad u, v \in \mathbb{R}^{2 N} .
$$

Close scrutiny reveals a natural bound for the ratios of eigenvalues of $A_{C} B_{C}^{-1}$ and the five-point finite difference matrix.

LEMMA 4.3. The eigenvalues $\lambda_{l}^{c}=\lambda_{l}\left(A_{C} B_{C}^{-1}\right), l= \pm 0, \pm 1, \ldots, \pm(2 N-1)$ defined in (4.8) and (4.9) satisfy

$$
4 \leq \frac{h^{2} \lambda_{l}^{c}}{\sin ^{2} \frac{\theta_{l}}{2}} \leq 9, \quad l=1,2, \ldots, 2 N-1 .
$$

Proof. It follows from Lemma 4.1 that for $1 \leq l \leq 2 N-1$,

$$
\frac{h^{2} \lambda_{l}^{c}}{\sin ^{2} \frac{\theta_{l}}{2}}=\frac{(7 \rho+9)-\sqrt{\rho^{2}+90 \rho+81}}{\left(\frac{2}{3} \rho+\frac{1}{2}\right) \sin ^{2} \frac{\theta_{l}}{2}}
$$

Letting

$$
G\left(\sin ^{2} \frac{\theta_{l}}{2}\right):=\frac{(7 \rho+9)-\sqrt{\rho^{2}+90 \rho+81}}{\left(\frac{2}{3} \rho+\frac{1}{2}\right) \sin ^{2} \frac{\theta_{l}}{2}}
$$

where $\rho=\frac{\sin ^{2} \frac{\theta_{l}}{2}}{1-\sin ^{2} \frac{\theta_{l}}{2}}$, for $0<x<1$ we have

$$
G^{\prime}(x)=-\frac{\sqrt{81-72 x-8 x^{2}}\left(\frac{2}{3} x^{2}-6 x-9\right)+\left(-\frac{8}{3} x^{3}-36 x^{2}+18 x+81\right)}{2\left(\frac{1}{6} x^{2}+\frac{1}{2} x\right)^{2} \sqrt{81-72 x-8 x^{2}}} \geq 0 .
$$

Thus,

$$
\max _{1 \leq l \leq 2 N-1} \frac{h^{2} \lambda_{l}^{c}}{\sin ^{2} \frac{\theta_{l}}{2}} \leq \lim _{x \rightarrow 1} G(x)=9
$$


and

$$
\min _{1 \leq l \leq 2 N-1} \frac{h^{2} \lambda_{l}^{c}}{\sin ^{2} \frac{\theta_{l}}{2}} \geq \lim _{x \rightarrow 0} G(x)=4 .
$$

From the above proof, we can see that the bounds in (4.15) are asymptotically optimal. Let

(4.17) $\quad w_{l}^{s}:=\left(\sin k \theta_{l}\right)_{k=0}^{2 N-1}, \quad$ and $\quad w_{l}^{c}:=\left(\cos k \theta_{l}\right)_{k=0}^{2 N-1}, \quad l=0,1,2, \ldots, 2 N-1$,

be $2 N$-dimensional vectors. If $A$ is a circulant matrix defined by

$$
A=\left(\begin{array}{ccccc}
a_{0} & a_{1} & & & a_{p} \\
a_{p} & a_{0} & a_{1} & & a_{p-1} \\
\cdots & \cdots & & & \\
a_{1} & a_{2} & & a_{p} & a_{0}
\end{array}\right)
$$

then it is well known [Dav79] that its eigenvalues are given by

$$
\lambda_{j}(A)=f\left(\epsilon_{j}\right), \quad j=0,1, \ldots, p,
$$

where

$$
f(x):=a_{0}+a_{1} x+\cdots+a_{p} x^{p}, \quad \epsilon_{j}:=e^{\frac{i 2 \pi j}{p+1}} .
$$

Furthermore, $A$ can be decomposed into

$$
A=F \Lambda(A) F^{*},
$$

where

$$
F:=\left(\epsilon_{j}^{k}\right)_{j, k=0}^{p} \quad \text { and } \quad \Lambda(A):=\operatorname{diag}\left(1, f\left(\epsilon_{1}\right), \ldots, f\left(\epsilon_{p}\right)\right) .
$$

LEMMA 4.4. a) If the $2 N \times 2 N$ circulant matrix $A$ is symmetric then

$$
\begin{aligned}
& A w_{j}^{c}=\lambda_{j}(A) w_{j}^{c} \\
& A w_{j}^{s}=\lambda_{j}(A) w_{j}^{s} .
\end{aligned}
$$

b) If $A$ is skew symmetric then

$$
\begin{aligned}
& A w_{j}^{c}=-\gamma_{j} w_{j}^{s} \\
& A w_{j}^{s}=\gamma_{j} w_{j}^{c},
\end{aligned}
$$

where $\gamma_{j}=\operatorname{Im}\left(f\left(\epsilon_{j}\right)\right)$.

Proof. Lemma 4.4a) is a straightforward calculation, noting the fact that

$$
F_{j}=w_{j}^{c}+i w_{j}^{s},
$$

where $F_{j}$ is the $j$ th column vector of $F$. By (4.19)

$$
A\left(w_{j}^{c}+i w_{j}^{s}\right)=f\left(\epsilon_{j}\right)\left(w_{j}^{c}+i w_{j}^{s}\right)
$$


Since each eigenvalue of a skew-symmetric circulant matrix is imaginary, $f\left(\epsilon_{j}\right)=i \gamma_{j}$, so (4.21) follows.

Let

$$
\begin{array}{ll}
u_{l}=\left(\begin{array}{c}
w_{l}^{s} \\
0
\end{array}\right), \quad u_{-N-l}=\left(\begin{array}{c}
0 \\
w_{N+l}^{s}
\end{array}\right), \quad l=1,2, \ldots, N-1, \\
u_{-l}=\left(\begin{array}{c}
0 \\
w_{l}^{c}
\end{array}\right), \quad u_{N+l}=\left(\begin{array}{c}
-w_{N+l}^{c} \\
0
\end{array}\right), \quad l=0,1, \ldots, N-1, \\
u_{0}=\left(\begin{array}{c}
e \\
0
\end{array}\right), \quad \text { and } \quad u_{-N}=\left(\begin{array}{c}
0 \\
e^{*}
\end{array}\right) .
\end{array}
$$

It follows directly that the eigenvectors of $A_{C} B_{C}^{-1}$ defined in (4.10)-(4.12) can be expressed by

$$
\left(v_{l}, v_{-l}\right)=\tilde{I}\left(u_{l}, u_{-l}\right) D_{l}
$$

where

$$
\tilde{I}=\frac{1}{2}\left(\begin{array}{cc}
I & -I \\
I & I
\end{array}\right), D_{l}=\left(\begin{array}{cc}
-z_{l} d_{l} & -z_{-l} d_{-l} \\
2 \tau h d_{l} & 2 \tau h d_{-l}
\end{array}\right), l=1, \ldots, 2 N-1, l \neq N
$$

$$
D_{0}=D_{N}=I_{2} \equiv\left(\begin{array}{cc}
1 & 0 \\
0 & 1
\end{array}\right)
$$

with

$$
d_{l}=\sqrt{\frac{-z_{-l}}{4 \tau^{2} h^{2}\left(z_{l}-z_{-l}\right)}}, \quad d_{-l}=\sqrt{\frac{z_{l}}{4 \tau^{2} h^{2}\left(z_{l}-z_{-l}\right)}}, \quad l=1,2, \ldots,(2 N-1), l \neq N .
$$

LEMMA 4.5. Each $D_{l}, l=0,1, \ldots, 2 N-1$, is an orthogonal matrix.

Proof. It is obvious that $D_{0} D_{0}^{T}=D_{N} D_{N}^{T}=I_{2}$. For $l=1,2, \ldots,(2 N-1), l \neq N$, by (4.24) we obtain

$$
D_{l} D_{l}^{T}=\left(\begin{array}{cc}
z_{l}^{2} d_{l}^{2}+z_{-l}^{2} d_{-l}^{2} & d_{l} d_{-l}\left(z_{l} z_{-l}+4 \tau^{2} h^{2}\right) \\
d_{l} d_{-l}\left(z_{l} z_{-l}+4 \tau^{2} h^{2}\right) & 4 \tau^{2} h^{2}\left(d_{l}^{2}+d_{-l}^{2}\right)
\end{array}\right) .
$$

It follows from (4.8) and (4.13) that

$$
z_{l} z_{-l}=\frac{16 \rho}{h^{2}}\left(-\frac{1}{\lambda_{l}}+\frac{h^{2}}{9}\right)\left(-\frac{1}{\lambda_{-l}}+\frac{h^{2}}{9}\right)=-\frac{h^{2}}{3}
$$

so

$$
z_{l} z_{-l}+4 \tau^{2} h^{2}=0
$$

Using (4.26) and (4.27), we obtain

$$
z_{l}^{2} d_{l}^{2}+z_{-l}^{2} d_{-l}^{2}=4 \tau^{2} h^{2}\left(d_{l}^{2}+d_{-l}^{2}\right)=1 .
$$

Thus,

$$
D_{l} D_{l}^{T}=I_{2}
$$


4.2. Bounds for condition numbers of preconditioned systems. The key to proving uniform boundedness of condition numbers of the preconditioned matrices $T$ in (4.6) is to show that $A_{C} B_{C}^{-1}$ and the preconditioner $A_{F}$ share $2 \mathrm{D}$ invariant subspaces.

THEOREM 4.1. Let $\left(A_{C}, B_{C}\right)$ define a Hermite cubic collocation discretization of (2.1) and (2.3) and $\left\{v_{l}\right\}_{l= \pm 0}^{ \pm(2 N-1)}$, defined in (4.10)-(4.12), be the eigenvectors of $A_{C} B_{C}^{-1}$. Then each $S_{l}=\operatorname{span}\left\{v_{l}, v_{-l}\right\}, l=0,1, \ldots, 2 N-1$, is an invariant subspace of $A_{F}$.

Proof. By (4.1) and (4.24), we have

$$
\tilde{I}^{T} A_{F} \tilde{I}=\frac{1}{2}\left(\begin{array}{cc}
(b+\alpha) I-\frac{1}{2}\left(C+C^{T}\right) & \frac{1}{2}\left(C^{T}-C\right) \\
\frac{1}{2}\left(C-C^{T}\right) & (b+\alpha) I+\frac{1}{2}\left(C+C^{T}\right)
\end{array}\right),
$$

where

$$
C+C^{T}=\left(\begin{array}{ccccc}
2 c & a & & & a \\
a & 2 c & a & & \\
\ldots & & & & \\
a & & & a & 2 c
\end{array}\right), \quad C-C^{T}=\left(\begin{array}{cccc}
0 & -a & & a \\
a & 0 & -a & \\
\cdots & & & \\
-a & & a & 0
\end{array}\right) .
$$

Thus,

$$
A_{F}\left(v_{l}, v_{-l}\right)=\tilde{I}\left(\begin{array}{cc}
(b+\alpha) I-\frac{1}{2}\left(C+C^{T}\right) & \frac{1}{2}\left(C^{T}-C\right) \\
\frac{1}{2}\left(C-C^{T}\right) & (b+\alpha) I+\frac{1}{2}\left(C+C^{T}\right)
\end{array}\right)\left(u_{l}, u_{-l}\right) D_{l} .
$$

It follows from Lemma 4.4 that

$$
\left\{\begin{array} { c } 
{ \frac { 1 } { 2 } ( C + C ^ { T } ) w _ { l } ^ { s } = ( c + a \operatorname { c o s } \theta _ { l } ) w _ { l } ^ { s } } \\
{ \frac { 1 } { 2 } ( C + C ^ { T } ) w _ { l } ^ { c } = ( c + a \operatorname { c o s } \theta _ { l } ) w _ { l } ^ { c } , }
\end{array} \quad \left\{\begin{array}{c}
\frac{1}{2}\left(C-C^{T}\right) w_{l}^{s}=-a \sin \theta_{l} w_{l}^{c} \\
\frac{1}{2}\left(C-C^{T}\right) w_{l}^{c}=a \sin \theta_{l} w_{l}^{s},
\end{array}\right.\right.
$$

and therefore,

$$
\begin{gathered}
A_{F}\left(v_{l}, v_{-l}\right)=\tilde{I}\left(u_{l}, u_{-l}\right)\left(\begin{array}{cc}
b+\alpha-c-a \cos \theta_{l} & -a \sin \theta_{l} \\
-a \sin \theta_{l} & b+\alpha+c+a \cos \theta_{l}
\end{array}\right) D_{l} \\
l=0,1, \ldots, 2 N-1 .
\end{gathered}
$$

Letting

$$
\bar{D}_{l}:=\left(\begin{array}{cc}
(b+\alpha)-\left(c+a \cos \theta_{l}\right) & -a \sin \theta_{l} \\
-a \sin \theta_{l} & (b+\alpha)+\left(c+a \cos \theta_{l}\right)
\end{array}\right)
$$

and using Lemma 4.5 and equation (4.23), (4.30) can be rewritten as

$$
A_{F}\left(v_{l}, v_{-l}\right)=\left(v_{l}, v_{-l}\right) D_{l}^{T} \bar{D}_{l} D_{l} .
$$

Thus, $S_{l}, l=0,1, \ldots, 2 N-1$, are invariant subspaces of $A_{F}$.

It remains to estimate the spectrum in each subspace $S_{l}$. The basic result is given in the theorem below; its detailed proof is in [SHR93].

THEOREM 4.2. Given $\left(A_{C}, B_{C}\right)$ and $A_{F}$ as in Theorem 4.1 with $\alpha>0$, there exist positive constants $C_{1}$ and $C_{2}$ independent of $h$ such that $T$ defined in (4.6) satisfies

$$
0<C_{1} \leq \lambda_{l}\left(T^{T} T\right) \leq C_{2}, \quad l=0,1, \ldots, 2 N-1 .
$$


Let $\kappa_{2}(T)$ denote the condition number of $T$ for the $L_{2}$ norm, i.e.,

$$
\kappa_{2}(T)=\frac{\max _{i} \lambda_{i}\left(T^{T} T\right)}{\min _{i} \lambda_{i}\left(T^{T} T\right)} .
$$

The two corollaries below follow immediately.

COROLLARY 4.1. There exists a constant $C_{1}^{*}$ independent of h such that

$$
\kappa_{2}(T) \leq C_{1}^{*}
$$

COROLlaRY 4.2. There exist constants $C_{3}^{*}$ and $C_{4}^{*}$ independent of $h$ such that

$$
0<C_{3}^{*} \leq \lambda_{l}(T) \leq C_{4}^{*}, \quad l=0,1, \ldots, 2 N-1,
$$

and

$$
\kappa_{1}(T) \leq \frac{C_{4}^{*}}{C_{3}^{*}}, \quad l=0,1, \ldots, 2 N-1 .
$$

Remarks. (i) Since each eigenvalue of $T=A_{F}^{-1}\left(A_{C} B_{C}^{-1}+\alpha I\right)$ is a continuous function of $\alpha$ in $(0, \infty)$ with

$$
\lim _{\alpha \rightarrow \infty} \lambda_{l}(T)=1 \text { and } \lim _{\alpha \rightarrow 0} \lambda_{l}(T)<\text { constant, } \quad l=0,1, \ldots, 2 N-1,
$$

$C_{1}$ and $C_{2}$ can be assumed to be independent of $\alpha$.

(ii) Theorem 4.2, while showing that the eigenvalues of the preconditioned matrix $T$ are bounded by constants, does not give an explicit bound. Numerical observation has shown that each $\lambda_{i}(T)$ decreases as $\alpha$ increases, with $\min \lambda_{i}(T)=\lim _{\alpha \rightarrow \infty} \lambda_{i}(T)=1$. Furthermore, for a given $\alpha>0$, the eigenvectors corresponding to both maximum and minimum eigenvalues of $T$ occur in the subspace $S_{0}$. A straightforward calculation gives [SHR93]

$$
\min \lambda_{i}(T)=1 \quad \text { and } \quad \max \lambda_{i}(T)=18 \tau-3+o(h) \approx 2.19615+o(h),
$$

which implies $\kappa_{1}(T)$ is approximately bounded by 2.196 . This agrees with the numerical results in $\S 2$ (see Table 2). The same relationships also hold in the case $\kappa_{2}(T)$.

When $\alpha=0, A_{F}$ is singular, so $T$ is undefined. We can then consider a generalized eigenvalue problem $A_{c} B_{c}^{-1} v=\lambda A_{F} v$. The eigenvalues corresponding to the subspace $S_{l}$ $(l>0)$ lie in $[1,18 \tau-3]$.

(iii) One can use central differences with a uniform mesh on $[0,1]$ instead of the nonuniform mesh $\left\{x_{i}^{*}\right\}$ in (2.3) and obtain a matrix having the same form as (4.5) with

$$
b=\frac{2}{(2 N+1)^{2}} \quad \text { and } \quad a=c=\frac{1}{(2 N+1)^{2}} .
$$

The above arguments hold with minor modification, and the boundedness of the eigenvalues of $T$ and $T^{T} T$ is still valid.

(iv) It is easy to find examples of some discretizations of PDEs for which the spectral condition numbers are bounded but the condition numbers in the $L_{2}$-norm are not. Nevertheless, some iterative methods can still be very effective and converge quickly when applied to such preconditioned systems if the eigenvalues are properly distributed.

It should be noted that the collocation system preconditioned by a simple finite difference scheme is a very special case. Since $A_{F}$ and $A_{C} B_{C}^{-1}$ have $2 N$ common invariant subspaces for a uniform mesh and periodic boundary conditions, both spectral condition numbers and 
condition numbers in the $L_{2}$-norm are bounded by constants independent of $h$. Our numerical results indicate that in the case of a nonuniform mesh and/or Dirichlet boundary conditions, the spectral condition numbers are bounded, although we have as yet been unable to provide a theoretical proof. Numerical results indicate that the corresponding condition numbers in the $L_{2}$-norm are unbounded [SHR93], however.

(v) Although the discussion is based on a 1D model problem, these theoretical results are straightforward to generalize for the corresponding 2D model problem.

5. Numerical solution of PDEs. The collocation matrices $A_{C}$ and $B_{C}$ are generally nonsymmetric, as are the resulting preconditioned matrices. A number of iterative methods have been developed in recent years for the solution of nonsymmetric preconditioned systems. They would be expected to be efficient in our context because of the relatively small condition numbers and the close concentration of eigenvalues for the preconditioned matrices. To illustrate that this is indeed the case, we consider two examples of $2 \mathrm{D}$ second-order elliptic PDEs defined on $\Omega=[0,2] \times[0,2]$ with Dirichlet boundary conditions. The orthogonal Hermite bicubic collocation method is used for the discretization of the PDEs. In order to avoid the effect of discretization errors in our tests, the right-hand sides and boundary conditions in the two examples are chosen such that

$$
u(x, y)=x y(2-x)(2-y)
$$

is both the PDE solution and the collocation solution. The five-diagonal row sum equivalence incomplete $\mathrm{LU}$ factorization $\left(H_{R S 5}\right)$ is a preconditioner and a generalized conjugate residual (GCR) method [EES83] is employed to solve the preconditioned collocation equations. The error is measured by

$$
e^{(k)}=\max _{i j}\left|u_{i j}^{(k)}-u_{i j}\right|,
$$

where $u_{i j}$ denotes the exact solution and its first and mixed derivative values at mesh points, and $u_{i j}^{(k)}$ denotes the corresponding numerical solution at the $k$ th iteration. For both examples, the initial guess is taken as zero. The iterative procedure is stopped when $e^{(k)}$ is smaller than a prescribed tolerance $\delta$.

Example 5.1 Poisson Equation.

$$
\Delta u=f(x, y) .
$$

In Table 5, we list the number of iterations required for preconditioned GCR (PGCR) with four tolerances and using the 2D Meshes 1-3. Since this example is separable, other iterative methods such as block Gauss-Seidel (BGS), block SOR (BSOR) [Sun94a], and ADC [Dyk87] are also applicable when a uniform mesh is used. The results with BGS and BSOR are also given in Table 5. ADC is not considered since it is actually a direct method for this simple problem [Dyk87]. Although the number of iterations for PGCR to achieve a prescribed tolerance increases with $N$, the growth rate is acceptable and is smaller than that of BGS or BSOR. For given $N$, PGCR requires nearly the same number of iterations for 2D Mesh 2 and 3 as for a uniform mesh.

Example 5.2 A Nonseparable Elliptic PDE (Prob. 18 in [RB85]).

$$
\frac{\partial^{2} u}{\partial x^{2}}+(1+x y) \frac{\partial^{2} u}{\partial y^{2}}+\cos (x) \frac{\partial u}{\partial x}-e^{-x} \frac{\partial u}{\partial y}+3 u=f(x, y) .
$$

None of other iterative methods are applicable for this nonseparable problem. Numbers of iterations for PGCR using various meshes and tolerances are summarized in Table 6. The same basic conclusions about PGCR can be made as in the previous example. 
TABLE 5

Example 5.1 Iteration numbers of $\left(H_{R S 5}\right)$ preconditioned GCR and (nonpreconditioned) BGS, BSOR methods.

\begin{tabular}{|c|c|c|c|c|c|c|}
\hline METHOD & MESH NO. & $\mathrm{N}$ & $\delta=10^{-3}$ & $\delta=10^{-4}$ & $\delta=10^{-5}$ & $\delta=10^{-6}$ \\
\hline \multirow{3}{*}{ PGCR } & 1 & 8 & 10 & 12 & 15 & 18 \\
\cline { 3 - 7 } & & 16 & 16 & 19 & 22 & 24 \\
\cline { 2 - 7 } & 2 & 8 & 10 & 13 & 15 & 17 \\
\cline { 2 - 7 } & & 16 & 17 & 19 & 22 & 24 \\
\cline { 2 - 7 } & \multirow{2}{*}{3} & 8 & 11 & 13 & 15 & 17 \\
\hline \multirow{2}{*}{ BGS } & 1 & 8 & 22 & 26 & 29 & 31 \\
\hline & & 16 & 65 & 29 & 37 & 44 \\
\hline BSOR & 1 & 8 & 9 & 13 & 125 & 154 \\
\cline { 3 - 7 } & & 16 & 17 & 22 & 28 & 16 \\
\hline
\end{tabular}

TABLE 6

Example 5.2 Iteration numbers of $\left(H_{R S 5}\right)$ preconditioned GCR method.

\begin{tabular}{|c|c|c|c|c|c|}
\hline MESH NO. & $\mathrm{N}$ & $\delta=10^{-3}$ & $\delta=10^{-4}$ & $\delta=10^{-5}$ & $\delta=10^{-6}$ \\
\hline \multirow{2}{*}{1} & 4 & 9 & 11 & 12 & 14 \\
\cline { 2 - 6 } & 8 & 12 & 14 & 16 & 17 \\
\cline { 2 - 6 } & 16 & 16 & 19 & 21 & 24 \\
\hline \multirow{3}{*}{2} & 4 & 8 & 10 & 12 & 13 \\
\cline { 2 - 6 } & 8 & 10 & 12 & 14 & 16 \\
\cline { 2 - 6 } & 16 & 16 & 19 & 23 & 25 \\
\hline \multirow{3}{*}{3} & 4 & 7 & 10 & 11 & 13 \\
\cline { 2 - 6 } & 8 & 8 & 11 & 13 & 16 \\
\cline { 2 - 6 } & 16 & 23 & 27 & 30 & 32 \\
\hline
\end{tabular}

6. Conclusions and comments. A technique has been presented to construct a finite difference preconditioning for orthogonal collocation matrices. Only cubic Hermite basis functions have been considered, although the preconditioning principles are straightforward to generalize for higher order collocation methods. It is shown numerically that the norms of eigenvalues of the preconditioned collocation matrices can be bounded below by one and above by a constant independent of the number of mesh nodes using the exact low-order finite difference preconditioner. For both smooth and moderately nonsmooth meshes, the preconditioned matrix has a small spectral condition number, with its eigenvalues located in a narrow interval on the positive real axis. As a consequence, conventional iterative methods such as Richardson and conjugate gradient iterations give fast convergence for the preconditioned collocation equations.

We have also investigated much cheaper alternatives involving incomplete LU factorizations based on a low-order finite difference approximation. The row sum equivalence version is particularly efficient although condition numbers may grow slowly with the number of mesh points. Moreover, the generalized conjugate residual method with this preconditioning has been seen to converge fast for both separable and nonseparable problems with uniform or nonuniform meshes.

While only limited experiments and comparisons have been performed, the results are quite encouraging. It is our intention to perform a theoretical analysis of these preconditioning schemes (possibly developing a general theoretical framework for the collocation methods analogous to what is done in [MP90] for the finite element method) and do a more careful comparison with the currently popular direct schemes for solving the collocation linear systems. 
Acknowledgment. The authors are indebted to Tom Manteuffel for his constructive suggestions.

\section{REFERENCES}

[AMR88] U. M. Ascher, R. M. MatTheis, AND R. D. Russell, Numerical Solution of Boundary Value Problems for Ordinary Differential Equations, Prentice-Hall, Englewood Cliffs, NJ, 1988.

[Ben91] K. R. BEnNETT, Parallel Collocation Methods for Boundary Value Problems, Ph.D. dissertation, University of Kentucky, Lexington, Kentucky, 1991.

[BFB92] B. BIALECKI, G. FAIRWEATHER, AND K. R. BENNETT, Fast direct solvers for piecewise Hermite bicubic orthogonal spline collocation equations, SIAM J. Numer. Anal., 29 (1992), pp. 156-173.

[Bia91] B. BIALECKI, An alternating direction implicit method for orthogonal spline collocation linear systems, Numer. Math., 59 (1991), pp. 413-429.

[CE88] T. F. CHAN AND H. C. ELMAN, Fourier analysis of iterative methods for elliptic problems, SIAM Rev., 31 (1988), pp. 20-49.

[CHQZ88] C. Canuto, M. Y. Hussaini, A. Quarteroni, And T. A. Zang, Spectral Methods in Fluid Dynamics, Springer-Verlag, Berlin, 1988.

[CP91] K. D. COOPER AND P. M. PRENTER, Alternating direction collocation for separable elliptic partial differential equations, SIAM J. Numer. Anal., 28 (1991), pp. 711-727.

[Dav79] P. J. DAvis, Circulant Matrices, Wiley, New York, 1979.

[DS73] C. DE Boor AND B. SwARTZ, Collocation at Gaussian points, SIAM J. Numer. Anal., 10 (1973), pp. 582-606.

[Dyk87] W. R. DYKSEN, Tensor product generalized ADI methods for separable elliptic problems, SIAM J. Numer. Anal., 28 (1987), pp. 59-76.

[EES83] C. EISENTAT, H. C. Elman, AND M. H. SCHUlz, Variational iterative method for nonsymmetric systems of linear equations, SIAM J. Numer. Anal., 20 (1983), pp. 345-357.

[Eva83] D. J. Evans (ed.), Preconditioning Methods: Analysis and Applications, Gordon and Breach, New York, 1983.

[Hay80] L. HAYES, An alternating direction collocation method for finite element approximations on rectangles, Comput. Math. Appl., 6 (1980), pp. 45-50.

[HPC81] L. HAYES, G. PINDER, AND M. CELIA, Alternating direction collocation for rectangular regions, Comput. Methods Appl. Mech. Engrg., 27 (1981), pp. 265-277.

[JMPW92] W. JOUBERT, T. A. MANTEUfFel, S. V. PARTER, AND S. WANG, Preconditioning second-order elliptic operators: experiment and theory, SIAM J. Sci. Statist. Comput., 13 (1992), pp. 259-288.

[MP90] T. A. MANTEUfFel AND S. V. PARTER, Preconditioning and boundary conditions, SIAM J. Numer. Anal., 27 (1990), pp. 656-694.

[Ors80] S. A. ORSZAG, Spectral methods for problems in complex geometries, J. Comput. Phys., 37 (1980), pp. 70-92.

[PR76] P. M. PRENTER AND R. D. RuSSELL, Orthogonal collocation for elliptic partial differential equations, SIAM J. Numer. Anal., 13 (1976), pp. 923-939.

[RB85] J. R. RICE AND R. F. BoISverT, Solving Elliptic Problem Using Ellpack, Springer-Verlag, New York, 1985.

[RS72] R. D. RuSSELl AND L. F. SHAMPINE, A collocation method for boundary value problems, Numer. Math., 19 (1972), pp. 1-28.

[SHR93] W. Sun, W. HuANG, AND R. D. Russell, Finite Difference Preconditioning for Solving Orthogonal Collocation Equations for BVPs, Research Report No.93-08, Department of Mathematics and Statistics, Simon Fraser University, Burnaby, Canada, 1993.

[Sun94a] W. Sun, Iterative algorithms for solving Hermite bicubic collocation equations, SIAM J. Sci. Comput., 16 (1995), pp. 720-737.

[Sun94b] _ A high order direct method for solving Poisson's equation on a disc, Numer Math., 70 (1995), pp. 501-506.

[SZ89] W. SUN AND N. G. ZAMANI, A fast algorithm for solving the tensor product collocation equations, J. Franklin Inst., 326 (1989), pp. 295-307.

[Won87] Y. S. WonG, Preconditioned gradient type methods applied to nonsymmetric linear systems, Internat. J. Comput. Math., 23 (1987), pp. 141-165. 